Research Article

\title{
Vibration-Based Fault Diagnosis of Commutator Motor
}

\author{
Adam Glowacz $\mathbb{D}$ and Witold Glowacz \\ AGH University of Science and Technology, Faculty of Electrical Engineering, Automatics, \\ Computer Science and Biomedical Engineering, Department of Automatic Control and Robotics, Al. A. Mickiewicza 30, 30-059, \\ Kraków, Poland \\ Correspondence should be addressed to Adam Glowacz; adglow@agh.edu.pl
}

Received 15 July 2018; Revised 8 September 2018; Accepted 27 September 2018; Published 24 October 2018

Academic Editor: Tony Murmu

Copyright (c) 2018 Adam Glowacz and Witold Glowacz. This is an open access article distributed under the Creative Commons Attribution License, which permits unrestricted use, distribution, and reproduction in any medium, provided the original work is properly cited.

\begin{abstract}
This paper presents a study on vibration-based fault diagnosis techniques of a commutator motor (CM). Proposed techniques used vibration signals and signal processing methods. The authors analysed recognition efficiency for 3 states of the CM: healthy $\mathrm{CM}, \mathrm{CM}$ with broken tooth on sprocket, CM with broken rotor coil. Feature extraction methods called MSAF-RATIO-50-SFC (method of selection of amplitudes of frequencies ratio 50 second frequency coefficient), MSAF-RATIO-50-SFC-EXPANDED were implemented and used for an analysis. Feature vectors were obtained using MSAF-RATIO-50-SFC, MSAF-RATIO-50-SFCEXPANDED, and sum of RSoV. Classification methods such as nearest mean (NM) classifier, linear discriminant analysis (LDA), and backpropagation neural network (BNN) were used for the analysis. A total efficiency of recognition was in the range of $79.16 \%-93.75 \%\left(T_{\mathrm{V}}\right)$. The proposed methods have practical application in industries.
\end{abstract}

\section{Introduction}

Commutator motors are essential for various industries. They are used for application of automobile motors such as electric generators, wiper motor, window lifting motor, vehicle starters, seat incliner, fuel pump, side view mirror, and air-conditioning. Commutator motors are also used for power tools applications, for example, drilling machine motor, circular saw motor, and hammer tool motor. They are used for home appliances such as washing machine motor, motor of vacuum cleaner, motor of printer, and hair dryer motor.

Unexpected failures of motors generate unexpected stops. It causes losses of production time and money. To avoid failures, engineers developed online condition monitoring of motors. Condition monitoring helps engineers to take diagnostic decision on the basis of measured signals. Fault diagnosis techniques can detect faults and provide diagnostic information about the motor. It also allows us to use the motor for a longer time. Vibrations signals depend on states of the commutator motor. Each fault is associated with vibration signals. This correlation between states of the motor and characteristic frequencies is essential for fault diagnosis.
The main task for fault diagnosis based on vibration analysis is to find the best method for proper condition monitoring.

Many scientific articles described fault diagnosis techniques and different faults of machinery [1-13]. The early fault state can be detected by early fault diagnostic methods. Sometimes, it takes 5 minutes to damage the motor, for example, short-circuit of stator windings. Other faults such as bearings, damaged sprocket, air-gap, bent shaft, and broken rotor bar take much more time to damage the motor permanently.

Condition monitoring of electric motors was developed for measurement and analysis of diagnostic signals such as acoustic [1, 2], thermal [3,4], electric current [5-7], and vibration [8-13]. Each type of signal has advantages and disadvantages. Measurements of acoustic signal and thermal signal are noninvasive. Acoustic and thermal signals can be measured without touching the motor. The disadvantage of mentioned diagnostic signals is difficult processing. A methodology based on the analysis of acoustic signals for faults of the induction motor was presented [1]. The presented technique used the complete ensemble empirical mode decomposition. Delgado-Arredondo et al. analysed the following faults: bearing defects, mechanical unbalance, 
and two broken rotor bars [1]. The proposed approach could be used to identify mentioned faults in the industry. Another approach based on acoustic analysis was developed by Islam et al. [2]. In [2], the authors proposed a diagnostic method of induction motors using Gabor filtering and MCSVMs. Average classification accuracy of the diagnostic method was equal to $99.80 \%$.

The thermal imaging camera is highly priced. Moreover, it takes time to diagnose temperature of the motor. It is also limited for electrical faults. However, industrial use of technique-based thermal imaging gained a noticeable attention [3]. Inter-turn faults and cooling system faults were analysed in [3]. The analysis was conducted for induction motors. Singh and Naikan proposed an algorithm using infrared thermography for diagnosis of induction motors. The authors developed two thermal profiles indicators. Developed profiles indicators were used for analysis of thermal distributions [3].

Several industrial examples for thermal analysis of electrical motors operating in a petrochemical plant were presented. The results showed that thermal imaging can be useful for transmission system faults, cooling system faults, defective connections, stator faults, and bearing failures [4].

Current monitoring is low-cost and reliable method of fault diagnosis. Motor current signature analysis (MCSA) is often used for current analysis. Usually MCSA provides good results [1, 5, 6]. Singh and Naikan [5] proposed a method using MUSIC analysis (MSC-MUSIC). The proposed method was analysed for broken rotor bar and half broken rotor bar faults of the induction motor. Surprisingly, MCSA was found to be ineffective to recognize half broken rotor bar fault properly [5]. In [6], the authors studied MCSA and ZSC (zero sequence current) methods for 4 induction motors. Antonino-Daviu et al. analysed the following states: healthy, broken bar, two nonadjacent broken bars, and two adjacent broken bars. The authors proved the usefulness of the ZSC method for detection of broken rotor bars. However, they noticed that MCSA had some problems to detect analysed faults [6]. Bazan et al. [7] described a current analysis for fault diagnosis of three-phase induction using ANN. Classification accuracy 99\% was obtained for analysed cases [7].

Vibration analysis is an effective and immediate fault diagnosis technique. Vibration signals are also acquired with low noise level (from environment or other machines). Vibration analysis is used for detection of mechanical and electrical faults such as rotor, stator faults, bearings, misalignment, and faults of gear transmission systems [1, 3, 8]. A review about fault diagnosis methods for gear transmission systems using vibration analysis was presented [8]. It described following methodologies: methods based on ICA, order tracking, sparse decomposition, EMD, and wavelet [8]. The CMFE (composite multiscale fuzzy entropy) and ESVM (ensemble support vector machines) were used for detection of rolling bearing faults [9]. Zheng et al. analysed the influence of parameters of the CMFE. The CMFE was employed to extract features of the vibration signals. Next, ESVM was used as a classifier. The proposed approach was applied to experimental data analysis. It indicated that the approach was effective for detection of different faults of rolling bearings [9]. Duan et al. [10] described the development of condition monitoring of rolling bearings using vibration analysis, acoustic analysis, oil analysis, temperature analysis, and ultrasonic analysis. The authors indicated that multisensors information fusion is the trend of development [10]. Zurita-Millan et al. [11] proposed a vibration signal prognosis methodology of the electromechanical system (kinematic chain). The proposed methodology was based on neurofuzzy modeling using the patterns of the vibrations signal. They proved that the RMS method is a proper feature for vibration analysis. They obtained the results with an error lower than $2 \%$, but they did not analyse other sources of information, such as temperature and stator current [11]. Lu et al. described vibration-based condition monitoring of motor bearings [12]. A wireless sensor networks were used for motor bearings. The wireless sensor network prototype was developed. They proved that the sampled data length of the proposed approach result in a decrease of over $80 \%$. The proposed approach can be useful for machinery installed in remote areas, for example, wind farms [12]. In [13], the authors conducted vibration analysis for the detection of motor damages. They analysed bearing currents. Analyses of bearing faults are useful for industrial users of inverted-fed motors. They proved that time-frequency analysis of vibration signal is useful source of information.

In proposed research, the authors developed vibrationbased fault diagnosis of the commutator motor (CM). Vibration signals of the CM (healthy CM, CM with broken tooth on sprocket, and CM with broken rotor coil) were not analysed in the literature, so the authors decided to conduct such analyses. The authors analysed recognition efficiency of the vibration signal for 3 states of the CM: healthy CM (Figure 1), CM with broken tooth on sprocket (Figure 2), and CM with broken rotor coil (Figure 3). The feature extraction methods called MSAF-RATIO-50-SFC (Method of Selection of Amplitudes of Frequencies Ratio 50 Second Frequency Coefficient), MSAF-RATIO-50-SFC-EXPANDED were implemented and used for the analysis. Feature vectors were obtained using MSAF-RATIO-50-SFC, MSAFRATIO-50-SFC-EXPANDED, and sum of RSoV. Classification methods such as nearest mean (NM) classifier, Linear discirminant analysis (LDA), and backpropagation neural network (BNN) were used for the analysis.

The proposed techniques have practical application in industries. The analysed total efficiency of recognition was in the range of $79.16 \%-93.75 \%\left(T_{\mathrm{V}}\right.$ - see Section 3$)$. Low cost of the measuring device and low cost of the computer are the advantages of vibration-based fault diagnosis. It is also noninvasive technique of fault diagnosis. The acquired results are similar to other proposed techniques of fault diagnosis $[1,11]$. In the paper, original methods of feature extraction-MSAF-RATIO-50-SFC and MSAF-RATIO-50SFC-EXPANDED were used for vibration signals. It was proved that the proposed technique can be used for diagnosis of the CM. It was also proved that states of the motor such as healthy CM, CM with broken tooth on sprocket, and $\mathrm{CM}$ with broken rotor coil can be diagnosed using proposed techniques. 


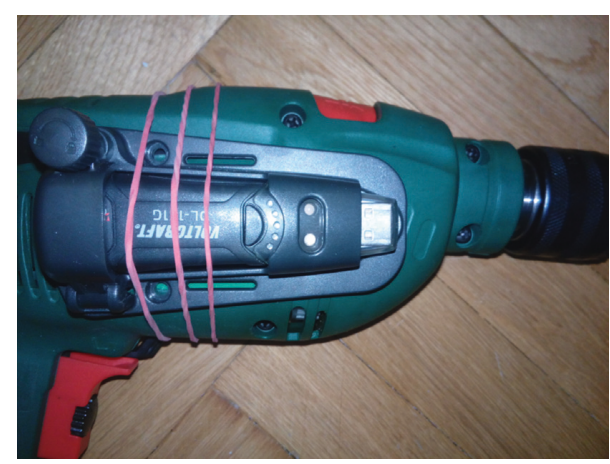

Figure 1: Healthy CM (rotor speed $3000 \mathrm{rpm}$ ).

\section{Proposed Vibration Fault Diagnosis Techniques}

The authors proposed 9 vibration diagnosis techniques (Figure 4). Techniques were based on signal processing methods and the step of acquisition of the vibration signal. The step of acquisition used a measuring device. The USB data logger Voltcraft DL-131G (sampling frequency $20 \mathrm{~Hz}$, 3 -axis recording) was used for the step of acquisition. Next computer software (Voltsoft Client) was used to download recorded data from the USB data logger. There are many types of acceleration USB data loggers and software. There were many possibilities of the step of acquisition of vibration signal. The first step of signal processing was split of measured vibration data. Vibration data were split into 5-second samples. Each 5-second sample had 100 values. The next step was feature extraction. Feature extraction was carried out using three different methods: MSAF-RATIO-50-SFC, MSAF-RATIO-50-SFC-EXPANDED, and sum of RSoV. Classification of computed vectors was the last step of vibration signal processing. Classification was carried out using pattern creation and testing. It was carried out using three different methods: NM classifier, LDA, and BNN.

\subsection{Method of Selection of Amplitudes of Frequencies Ratio 50} Second Frequency Coefficient. The MSAF-RATIO-50-SFC was based on FFT (fast Fourier transform) coefficients. The method computes features from the frequency spectrum. It can be noticed that the vibration signal was dependent on type of fault, type of machine, rotation speed, and size of machine. Steps of the MSAF-RATIO-50-SFC were as follows:

(1) Computation of FFT spectra of analysed vibration signals.

(2) Computation of differences (difference of frequency coefficient (DFC)) between computed FFT spectra of analysed states.

(3) Computation of a ratio RS for analysed differences. The ratio $\mathrm{RS}$ was defined as

$$
\mathrm{RS}=(100 \%) \frac{\mathrm{DFC}_{n}}{\mathrm{SDFC}_{\text {max }}},
$$

where $\mathrm{SDFC}_{\max }$ is the second maximal amplitude of analysed difference of the FFT spectrum and $\mathrm{DFC}_{n}$ is the amplitude of analysed difference with index $n$. If $\mathrm{RS}=50 \%$ for MSAF-RATIO-50-SFC, then amplitude greater than $50 \%$ of second maximal amplitude is analysed.

(4) Selection of frequency coefficients for ratio RS $=50 \%$.

(5) Selection of common frequency coefficients for all computed differences.

(6) Form a feature vector.

A flow diagram of the MSAF-RATIO-50-SFC is shown (Figure 5).

The authors analysed vibration signals for 3 states of the CM: healthy CM, denoted as hcm; CM with broken tooth on sprocket, denoted as btos; CM with broken rotor coil, denoted as brc. Differences (DFC) between computed FFT spectra of analysed states are depicted in Figures 6-8.

The MSAF-RATIO-50-SFC computed following (five) common frequencies: $0.2,5.6,7.2,7.8,8 \mathrm{~Hz}$. The computed frequency components were used for the feature vector. Next computed feature vectors were used for the classification step.

2.2. Method of Selection of Amplitudes of Frequencies Ratio 50 Second Frequency Coefficient EXPANDED. The MSAFRATIO-50-SFC-EXPANDED is similar to the MSAFRATIO-50-SFC. It used several training sets to compute common frequency components. Steps of the MSAFRATIO-50-SFC-EXPANDED were following:

(1) Computation of FFT spectra of analysed vibration signals.

(2) Computation of differences (difference of frequency Coefficient (DFC)) between computed FFT spectra of analysed states.

(3) Computation of the ratio RS for analysed differences. The ratio $R S$ was defined as follows:

$$
\mathrm{RS}=(100 \%) \frac{\mathrm{DFC}_{n}}{\mathrm{SDFC}_{\max }},
$$

where $\mathrm{SDFC}_{\max }$ is the second maximal amplitude of analysed difference of the FFT spectrum and $\mathrm{DFC}_{n}$ is the amplitude of analysed difference with index $n$. If $\mathrm{RS}=50 \%$ for MSAF-RATIO-50-SFC, then amplitude greater than $50 \%$ of second maximal amplitude is analysed.

(4) Selection of frequency coefficients for ratio RS $=50 \%$.

(5) Selection of parameter R-EXPANDED = (number of required common frequencies)/(number of all selected frequencies). The parameter R-EXPANDED was used to find the final number of common frequencies. Let us consider following example R-EXPANDED $=0.999$, in that case 3 of 3 frequencies are required $((3 / 3>0.999))$. If $\mathrm{R}-\mathrm{EXPANDED}=0.6$, the method required 2 of 3 frequencies $((2 / 3)>0.6)$ to select specific frequency component.

(6) Selection of common frequency coefficients for all computed differences using parameter R-EXPANDED. 


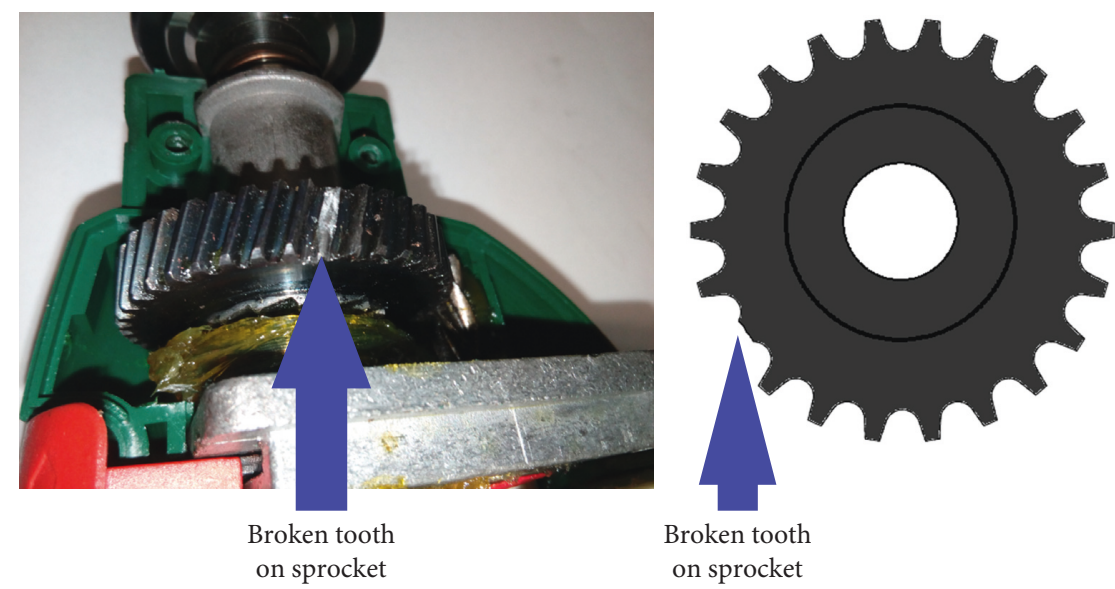

FIGURE 2: CM with broken tooth on sprocket.

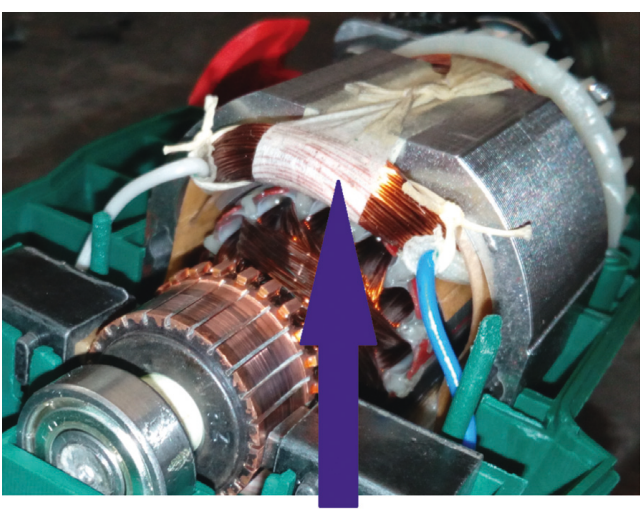

Broken rotor coil

(a)

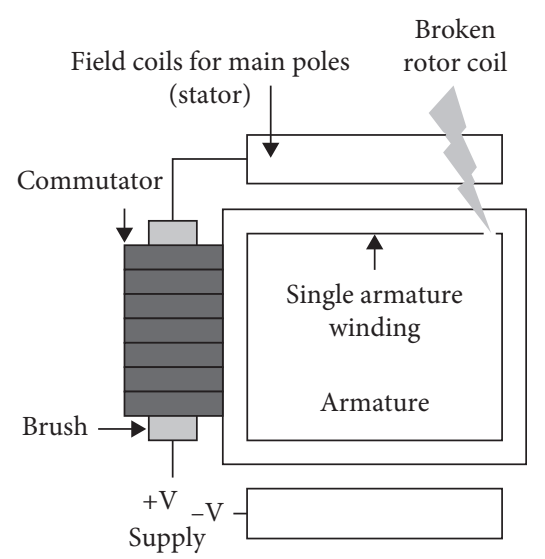

(b)

Figure 3: CM with broken rotor coil.

(7) Form a feature vector.

A flow diagram of the MSAF-RATIO-50-SFC-EXPANDED is shown (Figure 9).

The MSAF-RATIO-50-SFC-EXPANDED computed following (three) common frequencies: $0.2,7.4$, and $9 \mathrm{~Hz}$. The computed frequency components were used for the feature vector.

2.3. Feature Extraction Based on Sum of RSoV. Feature extraction based on sum of RSoV 1-element feature vectors. First, acceleration USB data logger measured: $\mathbf{X}$, $\mathbf{Y}$, and $\mathbf{Z}$ values and a resultant sum of vectors (RSoV). The resultant sum of vectors was expressed using the following formula:

$$
\mathrm{RSoV}=\sqrt[3]{|\mathbf{X}|^{3}+|\mathbf{Y}|^{3}+|\mathbf{Z}|^{3}}
$$

where $|\mathbf{X}|,|\mathbf{Y}|$, and $|\mathbf{Z}|$ are the lengths of $\mathbf{X}, \mathbf{Y}$, and $\mathbf{Z}$ vectors.

Measured five-second samples of vibration data had 100 values of RSoV. The measured values of RSoV were used to compute sum of RSoV. It was defined using the following formula:

$$
\text { Sum_of_RSoV }=\sum_{i=1}^{100}\left|\mathrm{RSoV}_{i}\right| \text {, }
$$

where $\mathrm{RSoV}_{i}$ is the resultant sum of vector with index $i$.

The authors presented 36 (1-element) feature vectors of vibration data consisting of sum of RSoV. It was presented in Table 1.

The computed values of feature vectors (sum of RSoV) are in the range $288.53-315\left(\mathrm{~m} / \mathrm{s}^{2}\right)$ for healthy CM, 298.08-349.67 $\left(\mathrm{m} / \mathrm{s}^{2}\right)$ for CM with broken rotor coil, and 891.13-947.04 $\left(\mathrm{m} / \mathrm{s}^{2}\right)$ for CM with broken tooth on sprocket. A problem of computed feature vectors was noticed. Feature vectors of healthy CM were similar to feature vectors of CM with broken rotor coil. The difficulty of classification is observed if the training sets of features are close to each other.

There are a lot of classification methods, for example, Neural networks, SVM [14, 15], LDA [16-18], nearest neighbor [19-21], rough sets [22, 23], and fuzzy classifiers [24]. The authors used 3 of them: NM classifier, LDA, and BNN.

2.4. Linear Discriminant Analysis (LDA). The authors used the LDA as a second method of data classification. Ronald 


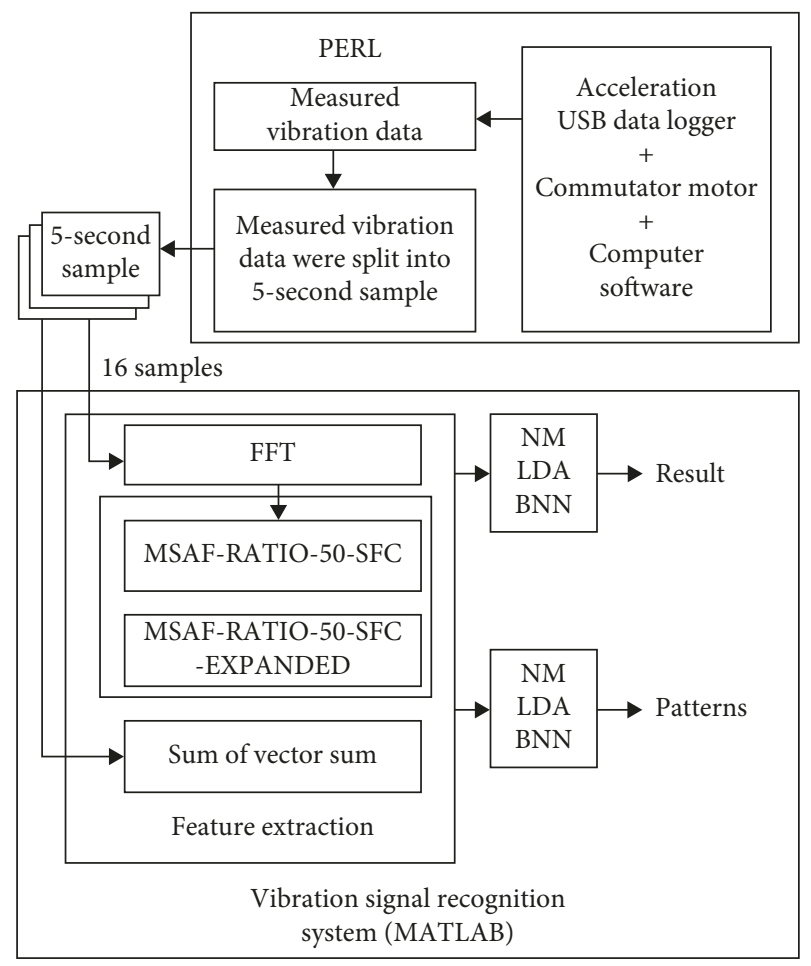

Figure 4: Proposed techniques using vibration signals and signal processing methods.

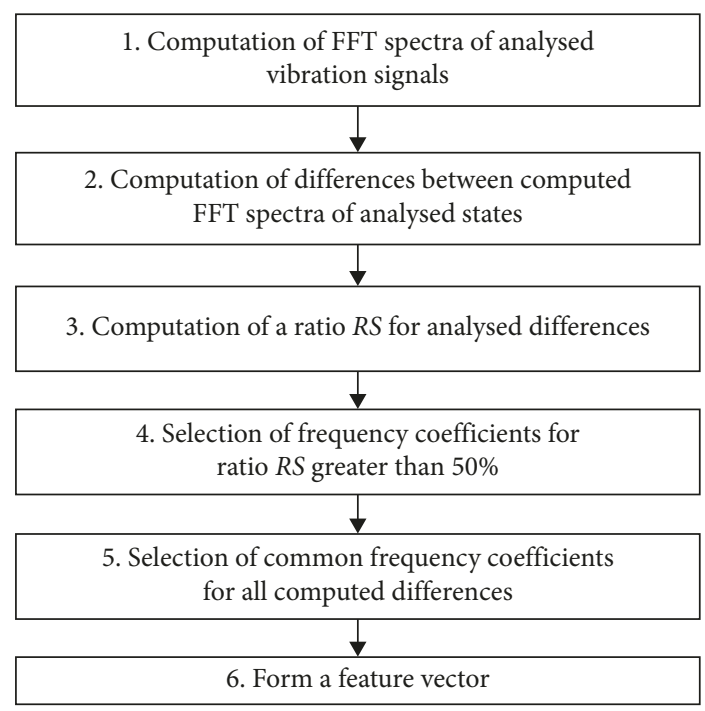

Figure 5: Flow diagram of the MSAF-RATIO-50-SFC.

Aylmer Fisher developed the LDA in 1936. Implementation of the LDA classifier was little time-consuming. The LDA also classified data for multiclass problems. It was used for many classification problems such as fault diagnosis [16-18], face recognition [25], and identification of cancer samples [26]. The LDA used the concept of searching for a linear combination of variables. It computed the score function. Next, it estimated the linear coefficients that maximize the score. Next, unknown test feature vector was classified. Vector was projected onto the maximally separating direction (smaller subspace).

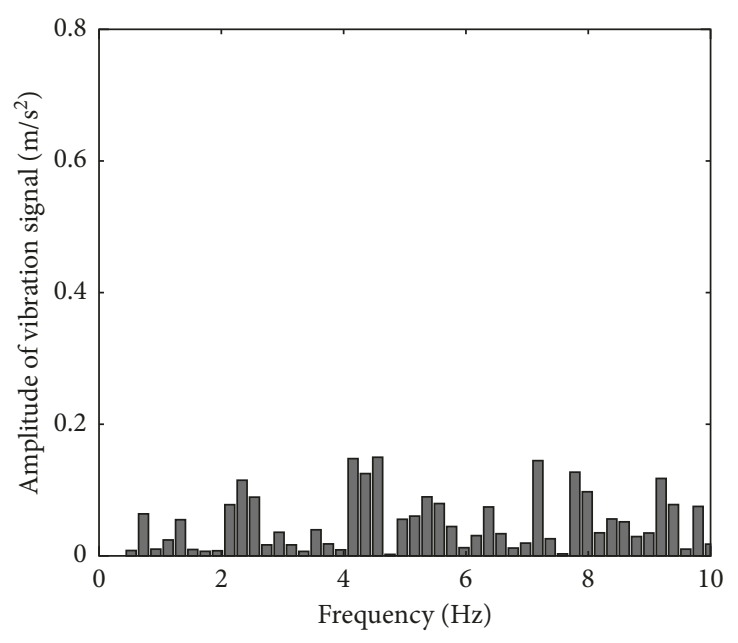

Figure 6: Difference (|hcm - brc|).

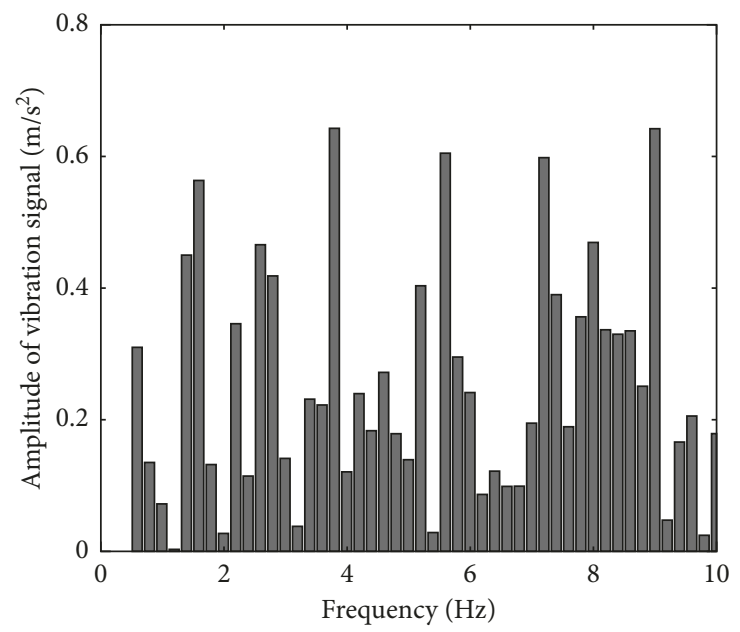

Figure 7: Difference (|hcm - btos $\mid)$.

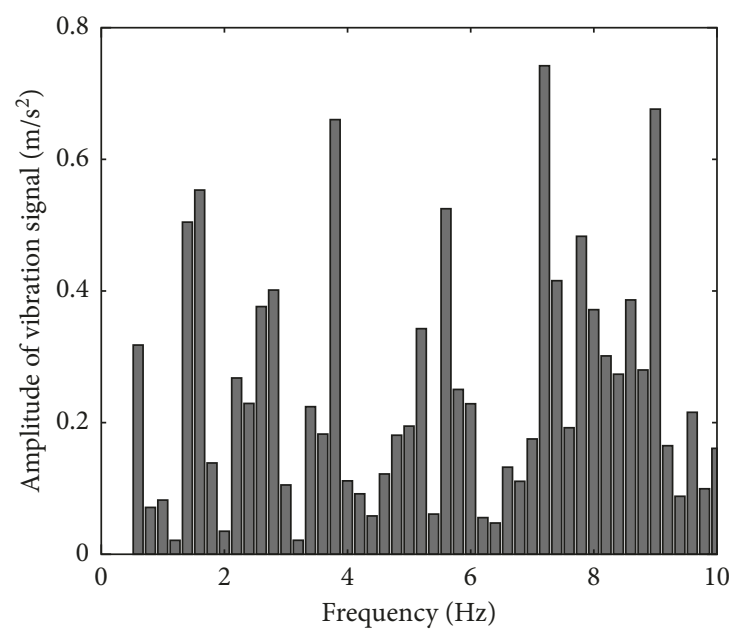

FIgURE 8: Difference (|brc - btos $\mid)$.

The steps of the LDA are presented in Figure 10. The LDA classifier was described more precisely in following articles [16-18, 25, 26]. 


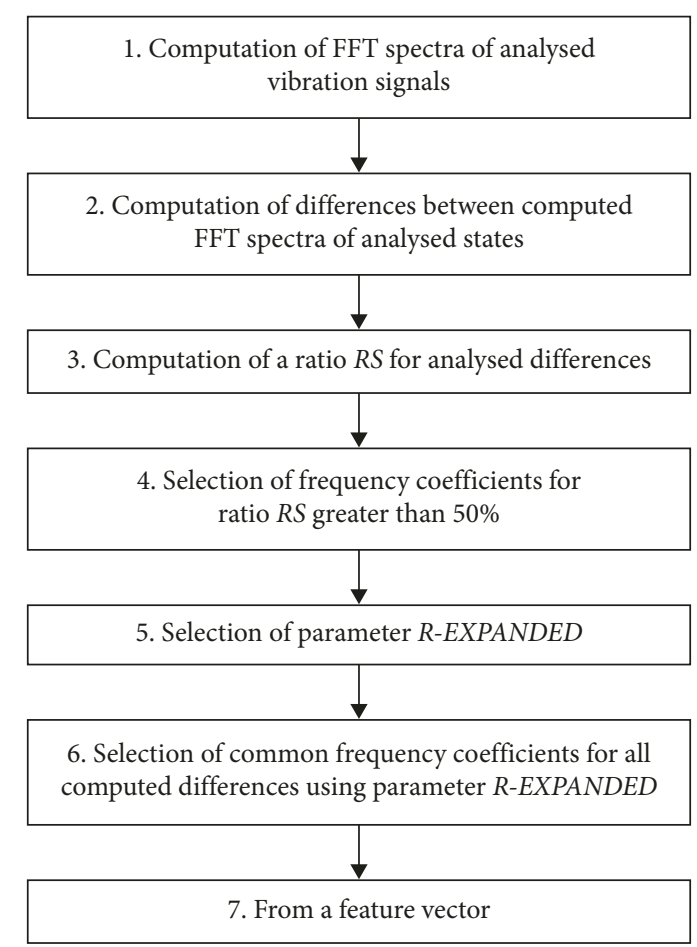

Figure 9: Flow diagram of the MSAF-RATIO-50-SFCEXPANDED.

2.5. Nearest Mean (NM). The nearest mean classifier used training average feature vector and test feature vector for data classification. Training average feature vector a was denoted as follows:

$$
\mathbf{a}=\frac{1}{n} \sum_{k=1}^{n} \mathbf{f}_{k}
$$

where $\mathbf{a}$ is training average feature vector, $\mathbf{f}$ is training feature vector, $n$ is the number of training feature vectors, and $\mathbf{f}_{k}$ is the value of training average feature vector with $k$ index $(k=5$ for MSAF-RATIO-50-SFC, $k=3$ for MSAF-RATIO-50-SFC-EXPANDED, and $k=1$ for sum of RSoV).

The NM classifier computed distance between the training average feature vector and test vector. It used distance function such as Manhattan distance or Euclidean distance. The authors used Manhattan distance:

$$
d(\mathbf{t}-\mathbf{a})=\sum_{i=1}^{k}\left|\left(t_{i}-a_{i}\right)\right|,
$$

where $d(\mathbf{t}-\mathbf{a})=$ distance, unknown test vector $\mathbf{t}=$ $\left[t_{1}, \ldots, t_{i}\right]$, and training average feature vector $\mathbf{a}=$ $\left[a_{1}, \ldots, a_{i}\right]$.

The classifier made decision about the class using the computed nearest distance. The NM classifier was described more precisely in following articles $[27,28]$.

2.6. Backpropagation Neural Network. The neural network based on the backpropagation method was the common supervised classification method. It used training and test sets of feature vectors. In the literatures [29-32], neural networks were used for fault diagnosis [29, 30], controlling a temperature field [31], prediction of speech quality [32], and classification of emotion recognition [33]. The authors used three-layer backpropagation neural network for data classification (input layer, hidden layer, and output layer). It was typical structure of the backpropagation neural network. The authors used following backpropagation neural network (Figure 11).

The input layer had 1,3 , or 5 neurons depending on feature extraction method. The hidden layer had 20 neurons. The output layer of BNN had 3 neurons. The values of output neurons were 001 , healthy CM, 010, CM with broken tooth on sprocket, and 100, CM with broken rotor coil.

\section{Results and Discussion}

Vibration signals of the CM were measured in a flat. The authors conducted analysis for 3 states of the CM: healthy CM (Figure 1), CM with broken tooth on sprocket (Figure 2), and CM with broken rotor coil (Figure 3 ). Parameters of the CM were $Q=1.84 \mathrm{~kg}, P=500 \mathrm{~W}, S=$ $3000 \mathrm{rpm}, V=230 \mathrm{~V}$, and $f=50 \mathrm{~Hz}$, where $Q$ is the weight of the CM motor, $P$ is the power of the CM motor, $S$ is the rotor speed of the CM motor, $V$ is the supply voltage of the CM motor, and $f$ is the current frequency of the CM motor.

Vibration signals were measured from $1 \mathrm{CM}$ (healthy $\mathrm{CM}, \mathrm{CM}$ with broken rotor coil, or $\mathrm{CM}$ with broken tooth on sprocket).

The authors used 9 training samples and 48 test samples of vibration signals (each sample has 5 seconds of vibration signal-100 measured values) for the analysis. Proposed fault diagnosis techniques (Figure 4) were used for signal processing of vibration signals. The evaluation of analysis of vibration signals was carried out using recognition efficiency of vibration signal $E_{\mathrm{V}}$. The value of $E_{\mathrm{V}}$ was expressed as follows:

$$
E_{\mathrm{V}}=\frac{N_{\text {recognized }}}{N_{\text {all }}} \cdot 100 \%,
$$

where $N_{\text {recognized }}$ is the number of test samples recognized for specific class, $N_{\text {all }}$ is the number of all test samples for specific class, and $E_{\mathrm{V}}$ is the recognition efficiency of vibration signal for specific class.

The total efficiency of vibration signal recognition was denoted as $T_{\mathrm{V}}$ follows:

$$
T_{\mathrm{V}}=\frac{E_{\mathrm{V} 1}+E_{\mathrm{V} 2}+E_{\mathrm{V} 3}}{3},
$$

where $E_{\mathrm{V} 1}$ is the $E_{\mathrm{V}}$ of the healthy $\mathrm{CM}, E_{\mathrm{V} 2}$ is the $E_{\mathrm{V}}$ of the $\mathrm{CM}$ with broken rotor coil, $E_{\mathrm{V} 3}$ is the $E_{\mathrm{V}}$ of the $\mathrm{CM}$ with broken tooth on sprocket, and $T_{\mathrm{V}}$ is the total $E_{\mathrm{V}}$ of analysed states of the CM.

The results are shown in Tables 2-10. In Table 2, the authors presented the results of recognition of vibration signals. The sum of RSoV (1 analysed feature) and the NM classifier were used.

In Table 3, the authors presented the results of recognition of vibration signals. The MSAF-RATIO-50-SFC (5 analysed features) and the NM classifier were used. 
TABLE 1: Sum of RSoV for 36 (1-element) feature vectors of vibration data.

\begin{tabular}{lccccc}
\hline Type of feature & \multicolumn{5}{c}{ Sum of RSoV $\left(\mathrm{m} / \mathrm{s}^{2}\right)$} \\
\hline Number of feature vectors & 1 & 2 & 3 & 4 & 5 \\
Healthy CM & 287.42 & 288.53 & 296.34 & 308.78 & 309.51 \\
Number of feature vectors & 7 & 8 & 9 & 10 & 11 \\
Healthy CM & 310.77 & 295 & 295.47 & 313.30 & 315 \\
Number of feature vectors & 13 & 14 & 15 & 16 & 12 \\
CM with broken rotor coil & 332.58 & 342.55 & 328.39 & 329.98 & 349.67 \\
Number of feature vectors & 19 & 20 & 21 & 22 & 31.01 \\
CM with broken rotor coil & 335.80 & 328.68 & 298.08 & 307.28 & 303.66 \\
Number of feature vectors & 25 & 26 & 27 & 28 \\
CM with broken tooth on sprocket & 947.04 & 915.37 & 917.93 & 940.20 & 29 \\
Number of feature vectors & 31 & 32 & 33 & 34.64 \\
CM with broken tooth on sprocket & 891.13 & 935.14 & 926.75 & 904.45 & 302.59 \\
\hline
\end{tabular}

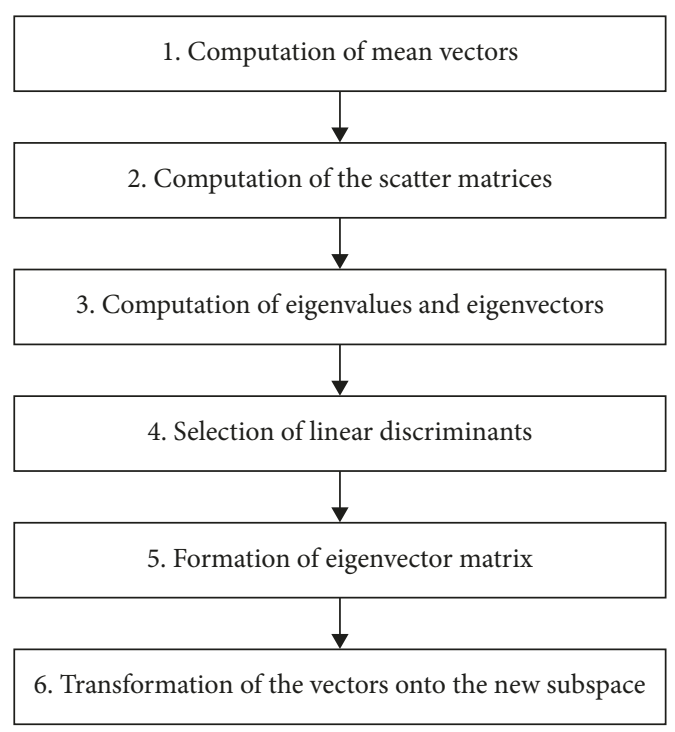

Figure 10: Steps of the LDA.

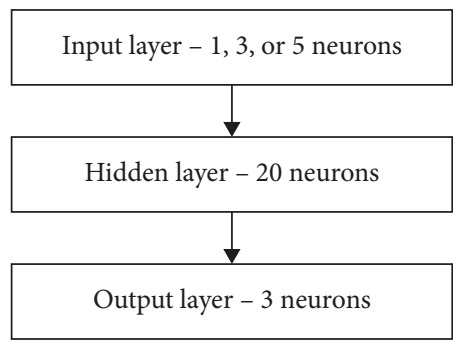

FIGURE 11: Structure of the proposed BNN.

In Table 4, the authors presented the results of recognition of vibration signals. The MSAF-RATIO-50-SFCEXPANDED (3 analysed features) and the NM classifier were used.

In Table 5, the authors presented the results of recognition of vibration signals. The sum of RSoV (1 analysed feature) and the LDA classifier were used.

In Table 6, the authors presented the results of recognition of vibration signals. The MSAF-RATIO-50-SFC (5 analysed features) and the LDA classifier were used.
TABLE 2: The results of recognition of vibration signals-the sum of RSoV and the NM classifier were used.

\begin{tabular}{lc}
\hline Type of vibration signal & $E_{\mathrm{V}}(\%)$ \\
\hline Healthy CM & 100 \\
CM with broken rotor coil & 81.25 \\
CM with broken tooth on sprocket & 100 \\
$T_{\mathrm{V}}$ & 93.75 \\
\hline
\end{tabular}

TABLE 3: The results of recognition of vibration signals-the MSAFRATIO-50-SFC and the NM classifier were used.

\begin{tabular}{lc}
\hline Type of vibration signal & $E_{\mathrm{V}}(\%)$ \\
\hline Healthy CM & 93.75 \\
CM with broken rotor coil & 81.25 \\
CM with broken tooth on sprocket & 100 \\
$T_{\mathrm{V}}$ & 91.66 \\
\hline
\end{tabular}

TABLE 4: The results of recognition of vibration signals-the MSAFRATIO-50-SFC-EXPANDED and the NM classifier were used.

\begin{tabular}{lc}
\hline Type of vibration signal & $E_{\mathrm{V}}(\%)$ \\
\hline Healthy CM & 93.75 \\
CM with broken rotor coil & 81.25 \\
CM with broken tooth on sprocket & 100 \\
$T_{\mathrm{V}}$ & 91.66 \\
\hline
\end{tabular}

TABLE 5: The results of recognition of vibration signals-the sum of RSoV and the LDA classifier were used.

\begin{tabular}{lc}
\hline Type of vibration signal & $E_{\mathrm{V}}(\%)$ \\
\hline Healthy CM & 93.75 \\
CM with broken rotor coil & 81.25 \\
CM with broken tooth on sprocket & 100 \\
$T_{\mathrm{V}}$ & 91.66 \\
\hline
\end{tabular}

In Table 7, the authors presented the results of recognition of vibration signals. The MSAF-RATIO-50-SFCEXPANDED ( 3 analysed features) and the LDA classifier were used.

In Table 8 , the authors presented the results of recognition of vibration signals. The sum of RSoV ( 1 analysed feature) and the backpropagation neural network were used. 
TABLE 6: The results of recognition of vibration signals-the MSAFRATIO-50-SFC and the LDA classifier were used.

\begin{tabular}{lc}
\hline Type of vibration signal & $E_{\mathrm{V}}(\%)$ \\
\hline Healthy CM & 75 \\
CM with broken rotor coil & 87.5 \\
CM with broken tooth on sprocket & 93.75 \\
$T_{\mathrm{V}}$ & 85.41 \\
\hline
\end{tabular}

TABLE 7: The results of recognition of vibration signals-the MSAFRATIO-50-SFC-EXPANDED and the LDA classifier were used.

\begin{tabular}{lc}
\hline Type of vibration signal & $E_{\mathrm{V}}(\%)$ \\
\hline Healthy CM & 81.25 \\
CM with broken rotor coil & 75 \\
CM with broken tooth on sprocket & 93.75 \\
$T_{\mathrm{V}}$ & 85.41 \\
\hline
\end{tabular}

In Table 9, the authors presented the results of recognition of vibration signals. The MSAF-RATIO-50-SFC (5 analysed features) and the backpropagation neural network were used.

In Table 10, the authors presented the results of recognition of vibration signals. The MSAF-RATIO-50-SFCEXPANDED (3 analysed features) and the backpropagation neural network were used.

The obtained results of analysed classifiers were in the range of $75 \%-100 \%\left(T_{\mathrm{V}}\right.$ was in the range of $79.16 \%-$ 93.75\%). Surprisingly, the sum of RSoV was the best feature for recognition of analysed states of the CM motor. It had $T_{\mathrm{V}}$ $=93.75 \%$ for the NM classifier. The MSAF-RATIO-50-SFC and the MSAF-RATIO-50-SFC-EXPANDED had a bit lower total efficiency $\left(T_{\mathrm{V}}\right)$. The authors compared the obtained results with other literature references. The acquired results were similar to other proposed techniques of vibration analysis [11, 20, 34-37]. In [11], results with an error lower than $2 \%$ were obtained. However, they [11] analysed bearing failure, 1/2-broken rotor bar, and 1 broken rotor bar of the induction motor. The article [34] presented a vibrationbased method for detection of bearing faults of the induction motor. Vibration signals were analysed using the FFT. The classification method was based on the SVM. Detection probability was in the range of 0.96-1 [34]. Another technique based on vibration signals was also used for diagnosis of the induction motor [35]. The proposed technique used the Shannon entropy. The $K$-means clustering method was used for classification. Recognition rate was $100 \%$ for healthy motor, half broken rotor bar, one broken rotor bar, and two broken rotor bars [35]. Learning features of vibration signals of the induction motor were analysed in the literature [36]. Deep Belief Network was used for the classification. Classification rate was in the range of 85.6-95.8\% [36]. Gangsar and Tiwari [37] presented vibration-based analysis for fault prediction of the induction motor. The MSVM (multiclass support vector machine) was used. It had average recognition rate in the range of 75-90\%. In [20], the authors also analysed vibration signals of the induction motor. The analysis was conducted using DWT and 3 classifiers: SVM, k-NN, and MLP. The best results were
TABLE 8: The results of recognition of vibration signals-the sum of $\mathrm{RSoV}$ and the backpropagation neural network were used.

\begin{tabular}{lc}
\hline Type of vibration signal & $E_{\mathrm{V}}(\%)$ \\
\hline Healthy CM & 93.75 \\
CM with broken rotor coil & 81.25 \\
CM with broken tooth on sprocket & 100 \\
$T_{\mathrm{V}}$ & 91.66 \\
\hline
\end{tabular}

TABLE 9: The results of recognition of vibration signals-the MSAFRATIO-50-SFC and the backpropagation neural network were used.

\begin{tabular}{lc}
\hline Type of vibration signal & $E_{\mathrm{V}}(\%)$ \\
\hline Healthy CM & 75 \\
CM with broken rotor coil & 87.5 \\
CM with broken tooth on sprocket & 75 \\
$T_{\mathrm{V}}$ & 79.16 \\
\hline
\end{tabular}

TABLE 10: The results of recognition of vibration signals-the MSAF-RATIO-50-SFC-EXPANDED and the backpropagation neural network were used.

\begin{tabular}{lc}
\hline Type of vibration signal & $E_{\mathrm{V}}(\%)$ \\
\hline Healthy CM & 75 \\
CM with broken rotor coil & 87.5 \\
CM with broken tooth on sprocket & 75 \\
$T_{\mathrm{V}}$ & 79.16 \\
\hline
\end{tabular}

obtained for the SVM (classification rate in the range of $98.8 \%-100 \%)$.

\section{Conclusions}

In this study, fault diagnostic techniques of the CM were developed. The developed techniques analysed vibration signals. The total efficiency of vibration signal recognition was analysed for 3 states of the CM: healthy CM, CM with broken tooth on sprocket, CM with broken rotor coil. Feature extraction methods MSAF-RATIO-50-SFC and MSAF-RATIO50-SFC-EXPANDED were implemented and used for fault diagnosis. Feature vectors were obtained using MSAFRATIO-50-SFC, MSAF-RATIO-50-SFC-EXPANDED, and sum of RSoV. Classification methods such as NM, LDA, and $\mathrm{BNN}$ were used for the analysis. The analysed total efficiency was in the range of $79.16 \%-93.75 \%\left(T_{\mathrm{V}}\right)$. The best feature for recognition was the sum of RSoV of analysed states of the CM motor. It had $T_{\mathrm{V}}=93.75 \%$ for the NM classifier.

Low cost of the measuring device (about 120\$) and low cost of the computer (about 270\$) are advantages of vibrationbased fault diagnosis. It is also noninvasive technique of fault diagnosis. Other faults such as bearing faults, rotor, and stator faults can be also diagnosed by analysis of vibration signals.

Future research will focus on development of new fault diagnosis techniques based on acoustic, vibration, and thermal signals. The authors will also analyse motors for different rotor speeds. New types of motors and faults will be analysed. The proposed techniques will be used for industries. 


\section{Data Availability}

The data used to support the findings of this study are available from the corresponding author upon request.

\section{Conflicts of Interest}

The authors declare that they have no conflicts of interest.

\section{Acknowledgments}

This work has been supported by AGH University of Science and Technology, grant nos. 11.11.120.714 and 11.11.120.815. We thank reviewers for their valuable suggestions.

\section{References}

[1] P. A. Delgado-Arredondo, D. Morinigo-Sotelo, R. A. OsornioRios, J. G. Avina-Cervantes, H. Rostro-Gonzalez, and R. D. Romero-Troncoso, "Methodology for fault detection in induction motors via sound and vibration signals," Mechanical Systems and Signal Processing, vol. 83, pp. 568-589, 2017.

[2] M. R. Islam, J. Uddin, and J. M. Kim, "Acoustic emission sensor network based fault diagnosis of induction motors using a Gabor filter and multiclass support vector machines," Ad Hoc \& Sensor Wireless Networks, vol. 34, no. 1-4, pp. 273-287, 2016.

[3] O. Janssens, R. Van de Walle, M. Loccufier, and S. Van Hoecke, "Deep learning for infrared thermal image based machine health monitoring," IEEE/ASME Transactions on Mechatronics, vol. 23, no. 1, pp. 151-159, 2018.

[4] D. Lopez-Perez and J. Antonino-Daviu, "Application of infrared thermography to failure detection in industrial induction motors: case stories," IEEE Transactions on Industry Applications, vol. 53, no. 3, pp. 1901-1908, 2017.

[5] G. Singh and V. N. A. Naikan, "Detection of half broken rotor bar fault in VFD driven induction motor drive using motor square current MUSIC analysis," Mechanical Systems and Signal Processing, vol. 110, pp. 333-348, 2018.

[6] J. A. Antonino-Daviu, K. N. Gyftakis, R. Garcia-Hernandez, H. Razik, and A. J. M. Cardoso, "Comparative influence of adjacent and non-adjacent broken rotor bars on the induction motor diagnosis through MCSA and ZSC methods," in Proceedings of IECON 2015-41st Annual Conference of the IEEE Industrial Electronics Society, pp. 1680-1685, IEEE Industrial Electronics Society, Yokohama, Japan, November 2015.

[7] G. H. Bazan, P. R. Scalassara, W. Endo, A. Goedtel, W. F. Godoy, and R. H. C. Palacios, "Stator fault analysis of three-phase induction motors using information measures and artificial neural networks," Electric Power Systems Research, vol. 143, pp. 347-356, 2017.

[8] Z. X. Li, Y. Jiang, C. Hu, and Z. Peng, "Recent progress on decoupling diagnosis of hybrid failures in gear transmission systems using vibration sensor signal: a review," Measurement, vol. 90, pp. 4-19, 2016.

[9] J. D. Zheng, H. Y. Pan, and J. S. Cheng, "Rolling bearing fault detection and diagnosis based on composite multiscale fuzzy entropy and ensemble support vector machines," Mechanical Systems and Signal Processing, vol. 85, pp. 746-759, 2017.

[10] Z. H. Duan, T. H. Wu, S. W. Guo, T. Shao, R. Malekian, and Z. X. Li, "Development and trend of condition monitoring and fault diagnosis of multi-sensors information fusion for rolling bearings: a review," International Journal of
Advanced Manufacturing Technology, vol. 96, no. 1-4, pp. 803-819, 2018.

[11] D. Zurita-Millan, M. Delgado-Prieto, J. J. Saucedo-Dorantes et al., "Vibration signal forecasting on rotating machinery by means of signal decomposition and neurofuzzy modeling," Shock and Vibration, vol. 2016, Article ID 2683269, 13 pages, 2016.

[12] S. L. Lu, P. Zhou, X. X. Wang, Y. B. Liu, F. Liu, and J. W. Zhao, "Condition monitoring and fault diagnosis of motor bearings using undersampled vibration signals from a wireless sensor network," Journal of Sound and Vibration, vol. 414, pp. 81-96, 2018.

[13] A. Prudhom, J. Antonino-Daviu, H. Razik, and V. ClimenteAlarcon, "Time-frequency vibration analysis for the detection of motor damages caused by bearing currents," Mechanical Systems and Signal Processing, vol. 84, pp. 747-762, 2017.

[14] M. T. Shervani-Tabar, M. M. Ettefagh, S. Lotfan, and H. Safarzadeh, "Cavitation intensity monitoring in an axial flow pump based on vibration signals using multi-class support vector machine," Proceedings of the Institution of Mechanical Engineers Part C-Journal of Mechanical Engineering Science, vol. 232, no. 17, pp. 3013-3026, 2018.

[15] M. Onel, C. A. Kieslich, Y. A. Guzman, C. A. Floudas, and E. N. Pistikopoulos, "Big data approach to batch process monitoring: simultaneous fault detection and diagnosis using nonlinear support vector achine-based feature selection," Computers \& Chemical Engineering, vol. 115, pp. 46-63, 2018.

[16] R. Z. Haddad and E. G. Strangas, "On the accuracy of fault detection and separation in permanent magnet synchronous machines using MCSA/MVSA and LDA," IEEE Transactions on Energy Conversion, vol. 31, no. 3, pp. 924-934, 2016.

[17] A. Yadav and A. Swetapadma, "A novel transmission line relaying scheme for fault detection and classification using wavelet transform and linear discriminant analysis," Ain Shams Engineering Journal, vol. 6, no. 1, pp. 199-209, 2015.

[18] A. Bazdar, R. B. Kazemzadeh, and S. T. A. Niaki, "Fault diagnosis within multistage machining processes using linear discriminant analysis: a case study in automotive industry," Quality Technology and Quantitative Management, vol. 14, no. 2, pp. 129-141, 2016.

[19] R. H. C. Palacios, I. N. da Silva, A. Goedtel, and W. F. Godoy, "A comprehensive evaluation of intelligent classifiers for fault identification in three-phase induction motors," Electric Power Systems Research, vol. 127, pp. 249-258, 2015.

[20] I. Cruz-Vega, J. Rangel-Magdaleno, J. Ramirez-Cortes, and H. Peregrina-Barreto, "Automatic progressive damage detection of rotor bar in induction motor using vibration analysis and multiple classifiers," Journal of Mechanical Science and Technology, vol. 31, no. 6, pp. 2651-2662, 2017.

[21] J. B. Xiong, Q. H. Zhang, Z. P. Peng, G. X. Sun, W. C. Xu, and Q. Wang, "A diagnosis method for rotation machinery faults based on dimensionless indexes combined with K-nearest neighbor algorithm," Mathematical Problems in Engineering, vol. 2017, Article ID 6872060, 2017.

[22] X. P. Yan, X. J. Xu, C. X. Sheng, C. Q. Yuan, and Z. X. Li, "Intelligent wear mode identification system for marine diesel engines based on multi-level belief rule base methodology," Measurement Science and Technology, vol. 29, no. 1, p. 015110, 2018.

[23] A. Krishnakumari, A. Elayaperumal, M. Saravanan, and C. Arvindan, "Fault diagnostics of spur gear using decision 
tree and fuzzy classifier," International Journal of Advanced Manufacturing Technology, vol. 89, no. 9-12, pp. 3487-3494, 2017.

[24] A. Mollajan, H. Memarian, and M. Nabi-Bidhendi, "Fuzzy classifier fusion: an application to reservoir facies identification," Neural Computing and Applications, vol. 30, no. 3, pp. 825-834, 2018.

[25] F. Damayanti, W. Setiawan, S. Herawati, and A. Rachmad, "Face recognition based on gender using a modified method of 2D-linear discriminant analysis," Advanced Science Letters, vol. 23, no. 12, pp. 12349-12353, 2017.

[26] C. L. M. Morais and K. M. G. Lima, "Principal component analysis with linear and quadratic discriminant analysis for identification of cancer samples based on mass spectrometry," Journal of the Brazilian Chemical Society, vol. 29, no. 3, pp. 472-481, 2018.

[27] G. Sergioli, E. Santucci, L. Didaci, J. A. Miszczak, and R. Giuntini, "A quantum-inspired version of the nearest mean classifier,” Soft Computing, vol. 22, no. 3, pp. 691-705, 2018.

[28] M. Loog and A. C. Jensen, "Semi-supervised nearest mean classification through a constrained log-likelihood," IEEE Transactions on Neural Networks and Learning Systems, vol. 26, no. 5, pp. 995-1006, 2015.

[29] Z. Y. Wang, C. Lu, and B. Zhou, "Fault diagnosis for rotary machinery with selective ensemble neural networks," $M e$ chanical Systems and Signal Processing, vol. 113, pp. 112-130, 2018.

[30] M. Simsir, R. Bayjr, and Y. Uyaroglu, "Real-time monitoring and fault diagnosis of a low power hub motor using feedforward neural network," Computational Intelligence and Neuroscience, vol. 2016, Article ID 7129376, 13 pages, 2016.

[31] H. Liu, J. Z. Zhou, Y. H. Xu, Y. Zheng, X. L. Peng, and W. Jiang, "Unsupervised fault diagnosis of rolling bearings using a deep neural network based on generative adversarial networks," Neurocomputing, vol. 315, pp. 412-424, 2018.

[32] L. Orcik, M. Voznak, J. Rozhon et al., "Prediction of speech quality based on resilient backpropagation artificial neural network," Wireless Personal Communications, vol. 96, no. 4, pp. 5375-5389, 2017.

[33] A. Turnip, A. I. Simbolon, M. F. Amri, P. Sihombing, R. H. Setiadi, and E. Mulyana, "Backpropagation neural networks training for EEG-SSVEP classification of emotion recognition," Internetworking Indonesia, vol. 9, no. 1, pp. 53-57, 2017.

[34] D. H. Hwang, Y. W. Youn, J. H. Sun, K. H. Choi, J. H. Lee, and Y. H. Kim, "Support vector machine based bearing fault diagnosis for induction motors using vibration signals," Journal of Electrical Engineering \& Technology, vol. 10, no. 4, pp. 1558-1565, 2015.

[35] D. Camarena-Martinez, M. Valtierra-Rodriguez, J. P. AmezquitaSanchez, D. Granados-Lieberman, R. J. Romero-Troncoso, and A. Garcia-Perez, "Shannon entropy and k-means method for automatic diagnosis of broken rotor bars in induction motors using vibration signals," Shock and Vibration, vol. 2016, Article ID 4860309, 10 pages, 2016.

[36] S. Y. Shao, W. J. Sun, P. Wang, R. X. Gao, and R. Q. Yan, "Learning features from vibration signals for induction motor fault diagnosis," in Proceedings International Symposium on Flexible Automation (ISFA), pp. 71-76, Cleveland, OH, USA, August 2016.

[37] P. Gangsar and R. Tiwari, "Comparative investigation of vibration and current monitoring for prediction of mechanical and electrical faults in induction motor based on multiclass-support vector machine algorithms," Mechanical Systems and Signal Processing, vol. 94, pp. 464-481, 2017. 


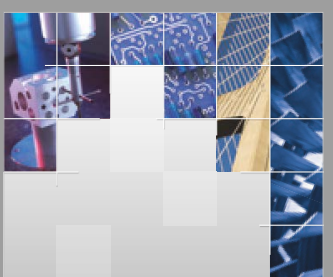

\section{Enfincering}
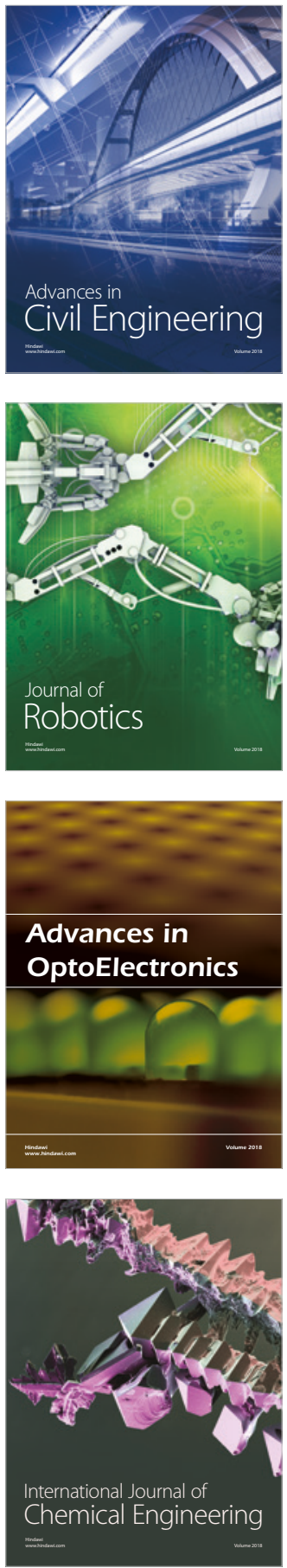

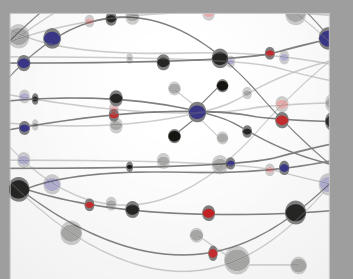

\section{Rotating \\ Machinery}

The Scientific World Journal

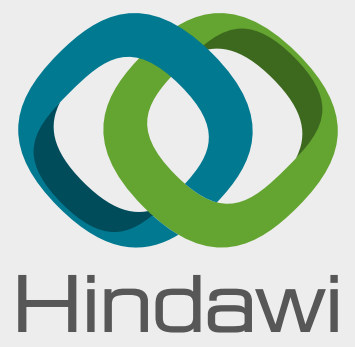

Submit your manuscripts at

www.hindawi.com
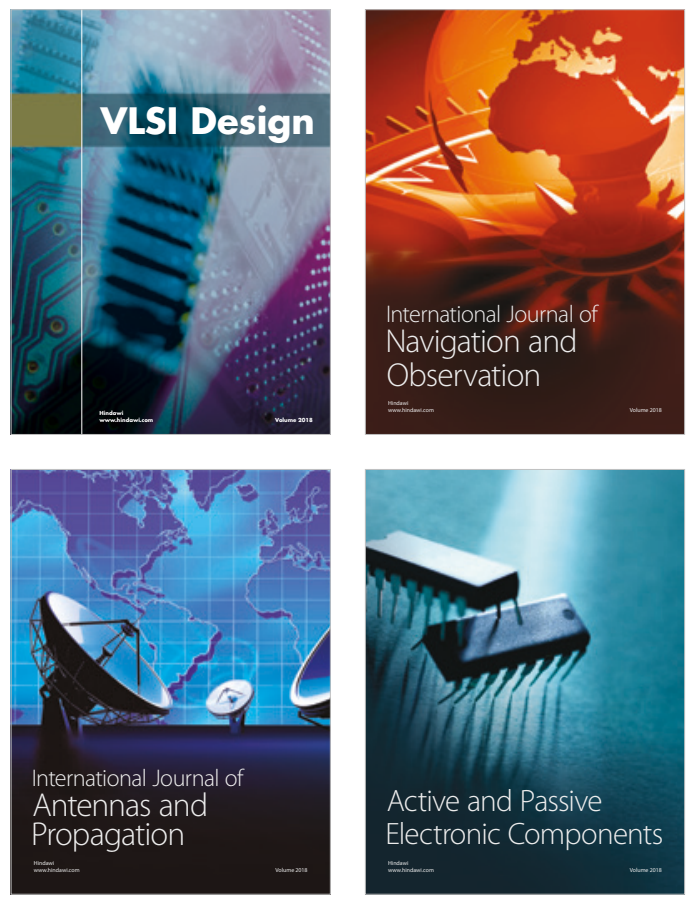
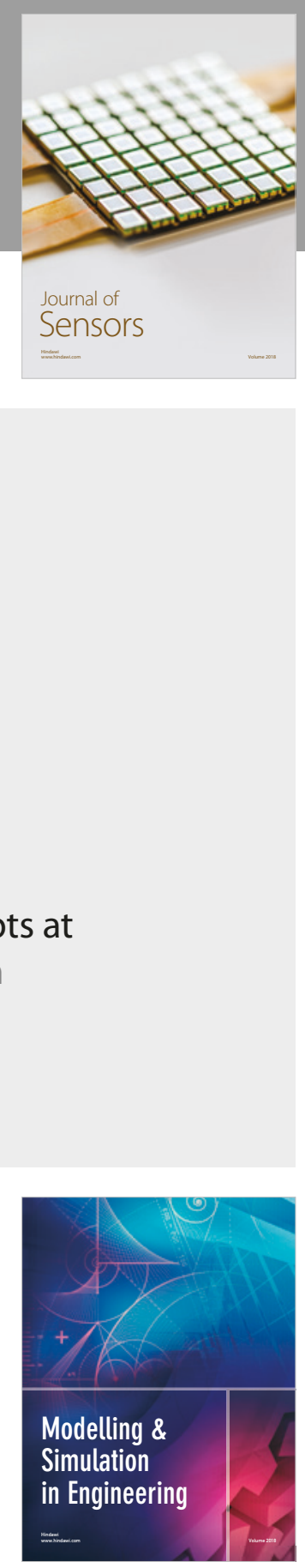

\section{Advances \\ Multimedia}
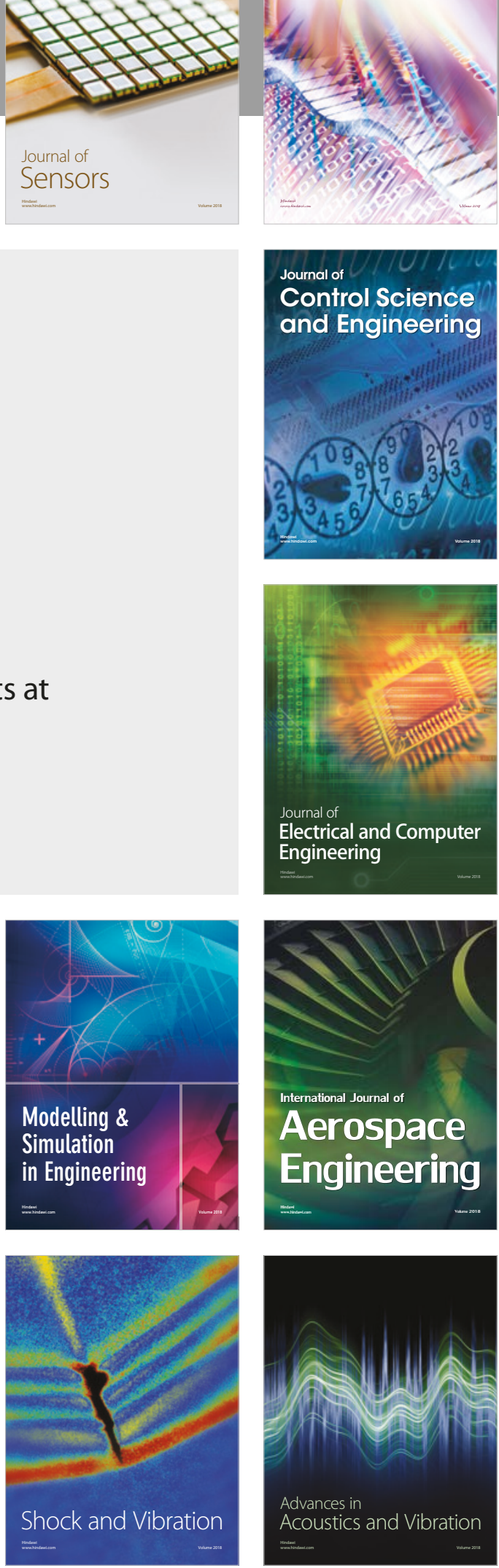